\title{
Prevention Methods of Posttraumatic Stress Disorder (PTST) in Cancer Survivors
}

\author{
Mohammadreza rezaei $^{1}$ and Nouraddin Abdi Goushbolagh ${ }^{2 *}$ \\ ${ }^{1}$ Department of Psychology, Faculty of Humanities, Islamic Azad University of Ahar, Iran \\ ${ }^{2}$ Department of Medical Physics, Faculty of Medicine, shahid sadoughi University of Medical Science, Iran \\ *Corresponding author: Nouraddin Abdi Goushbolagh, Medical Physics Department, Faculty of Medicine, shahid sadoughi University \\ of Medical Science, Iran
}

Published: 眥 October 30, 2019

\section{Introduction}

It is quite appropriate to say, people are less likely to look for diagnostic ways of cancer because of the fear of having cancer. In addition, many people have phobias of cancer treatment methods (such as surgery, chemotherapy, and radiotherapy) [1,2]. The fact remains that, many people are unwittingly exposed to the posttraumatic stress disorder (PTST) because of severe stress [3]. Nevertheless, many researchers do not believe to the psychology complications of cancer, but psychological distress during and after cancer treatment increased concerns about sexuality, intimacy, and physical well-being. These disorders can occur after the stressor agent such as cancer [4]. It is widely supposed that PTSD is psychological disease and separate from physiological system. Whereas, PTSD can have physiological consequences such as elevated blood pressure, cholesterol, and cortisol levels [4]. However, disabling subsyndromal PTSD symptoms is not known for each individual, but the consequences of this disorder make it more important to treat it $[4,5]$. Distress and anxiety and less optimal quality of life are caused by PTSD in cancer survivor. It would be better to say that PTSD has a direct effect on the quality of cancer treatment. As detailed, patient's spirit has a great impact on the positive response of cancer to chemical drugs and radiation doses during treatment. Also, treatment conditions can get worse PTSD. So that, the treatment environment, the attitude of the treatment technicians plays an important role in the recovery and doesn't involve them to PTSD. Passing on now to treatment methods of PTSD, we should try to suppress PTSD and comorbid symptoms by utilize treatment methods. Variety of psychotherapy (such as eye movement desensitization and reprocessing and cognitive restructuring method) and pharmacotherapy (such as prazosin, anticonvulsants and risperidone) that have been practiced on patients who suffer from PTSD symptoms induced by cancer that we will discuss below. Prazosin Utilize in PTSD is effective, particularly in reducing nightmares and improving sleep. PTSD is often associated with alcohol misuse, prazosin can reduce alcohol dependence [6].
Anticonvulsants have some beneficial attributes in treatment PTSD, particularly where irritability and a startle response are prominent [7]. Risperidone is associated with improvement in overall PTSD symptoms and specific sleep variables [8]. Some resources presented that benzodiazepines (BZDs) are treatment drugs for PTSD [9], But to be honest BZDs should be considered relatively contraindicated for patients with PTSD. Because it caused to worse psychotherapy outcomes, aggression, depression, and substance use [10]. Eye movement desensitization and reprocessing (EMDR) is faster and more effective psychotherapy method than other treatments. This method is a complex treatment that incorporates many different interventions, including imaginal exposure and free association [11]. Cognitive restructuring method included socratic questioning, guided discovery, the devil's advocate technique and determining the pros and cons of the validity of the assumption. Also, it has vital role for the effectiveness of the intervention [12]. Patients follow up after treatment is one of the most important in radiotherapy. Important considerate to the PTSD symptoms besides clinical examination after cancer treatment can help improved patient cancer. However, the emergence of PTSD can have a direct effect on the cancer treatment benefits. Thereby PTSD can pose a big challenge for cancer treatment researchers. The importance of this issue could provide the basis for a new collaboration between psychologists and oncologists to treat cancer with high therapeutic benefit without side effects.

\section{References}

1. Mundy EA, Blanchard EB, Cirenza E, Gargiulo J, Maloy B, et al. (2000) Posttraumatic stress disorder in breast cancer patients following autologous bone marrow transplantation or conventional cancer treatments. Behaviour Research and Therapy 38(10): 1015-1027.

2. Abdi Goushbolagh N, Keshavarz M, Zare MH, Bahreyni Toosi MH, Kargar $\mathrm{M}$, et al. (2019) Photosensitizer effects of MWCNTs $\mathrm{COOH}$ particles on CT26 fibroblastic cells exposed to laser irradiation. Artificial cells, nanomedicine, and biotechnology 47(1): 1326-1334. 
3. Luecken LJ, Dausch B, Gulla V, Hong R, Compas BE (2004) Alterations in morning cortisol associated with PTSD in women with breast cancer Journal of Psychosomatic Research 56(1): 13-15.

4. Hahn EE, Hays RD, Kahn KL, Litwin MS, Ganz PA (2015) Post traumatic stress symptoms in cancer survivors: Relationship to the impact of cancer scale and other associated risk factors. Psycho Oncology 24(6): 643-652.

5. Shelby RA, Golden Kreutz DM, Andersen BL (2008) PTSD diagnoses, subsyndromal symptoms, and comorbidities contribute to impairments for breast cancer survivors. Journal of Traumatic Stress: Official Publication of The International Society for Traumatic Stress Studies 21(2): 165-172.

6. Green B (2014) Prazosin in the treatment of PTSD. Journal of Psychiatric Practice 20(4): 253-259.

7. Ford N (1996) The use of anticonvulsants in posttraumatic stress disorder: Case study and overview. Journal of traumatic stress 9(4): 857-863.

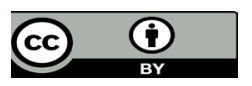

This work is licensed under Creative

Commons Attribution 4.0 License

To Submit Your Article Click Here:

Submit Article

DOI: $10.32474 /$ SJPBS.2019.03.000156
8. David D, De Faria L, Mellman TA (2006) Adjunctive risperidone treatment and sleep symptoms in combat veterans with chronic PTSD. Depression and anxiety 23(8): 489-491.

9. Berg A, Breslau N, Goodman S, Lezak M, Matchar D, et al. (2008) Treatment of PTSD: An assessment of the evidence. National Academies Press, Washington DC, USA.

10. Guina J, Rossetter SR, DeRHODES BJ, Nahhas RW, Welton RS (2015) Benzodiazepines for PTSD: A systematic review and meta-analysis. Journal of Psychiatric Practice 21(4): 281-303.

11. Taylor S, Thordarson DS, Maxfield L, Fedoroff IC, Lovell K, et al. (2003) Comparative efficacy, speed, and adverse effects of three PTSD treatments: Exposure therapy, EMDR, and relaxation training. Journal of consulting and clinical psychology 71(2): 330-338.

12. Müller Engelmann M, Steil R (2017) Cognitive restructuring and imagery modification for posttraumatic stress disorder (CRIM-PTSD): A pilot study. Journal of behavior therapy and experimental psychiatry 54: 44-50.

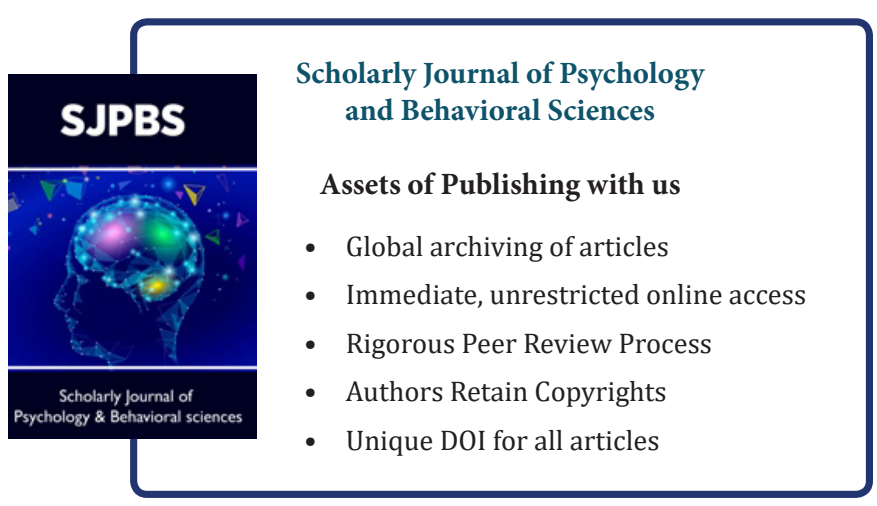

